\title{
DE L'ANTHROPOCÈNE À LA COLLAPSOLOGIE : REVALORISER LE CONTRAT NATUREL
}

\author{
Author(s) / Auteur(s) : \\ Prof. Damien CLAEYS \\ Faculté d'architecture, d'ingénierie architecturale, d'urbanisme (LOCI) \\ Laboratoire Théorie des systèmes en architecture (tsa-lab) \\ Université catholique de Louvain (UCLouvain) \\ damien.claeys@uclouvain.be
}

\begin{abstract}
Résumé :
À l'ère qu'ils nomment anthropocène, les scientifiques envisagent sérieusement l'effondrement imminent de notre civilisation à l'issue d'un crash global - qui pourrait prendre plusieurs années - affectant les dimensions écologique, sociétale et humaine. Cette conception objectivée du réel dans lequel nous vivons est le résultat d'une succession de travaux scientifique remontant, au moins, à la fin des années 1960 qui n'ont cessés d'avertir la société des effets négatifs et prévisibles d'une surconsommation des ressources planétaires, d'une fragilisation des liens sociétaux entre les êtres humains et des mises sous contrainte psychologique des leurs cerveaux.

Un parallélisme apparaît entre l'origine de cette prise de conscience et celle des modélisations pour comprendre les systèmes naturels et humains à l'aide de la théorie des systèmes, pensée relationnelle des phénomènes observés intégrant ces derniers dans leur contexte avant de les étudier.

Aujourd'hui, confronté aux effets d'une crise mondialisée, le fonctionnement global des sociétés humaines repose encore sur l'exploitation de la biocapacité planétaire et la stratification des classes sociales. La pensée systémique est l'une des méthodes capable de modéliser leur fonctionnement de manière à pouvoir les transformer en profondeur.
\end{abstract}

\section{Keywords / Mots-clés :}

système, écosystème, modèle, effondrement, décroissance, soutenabilité, responsabilité, anthropocène, capitalocène, collapsologie

Peinte par Jules Romain pour le duc Frédéric II de Mantoue, la fresque La chute des Géants (15321534) du palais du Té est une excellente allégorie de l'état actuel présumé de notre civilisation, menacé par l'imminence d'un crash global, affectant en même temps les dimensions écologique, sociétale et humaine (Claeys \& Lambert, 2018). Elle éclaire à sa manière les situations de crise contemporaines. Le thème mythologique lui-même donne corps aux préoccupations écologiques actuelles puisque la déesse Gaïa [la terre] est, à la fois, une génitrice - celle qui engendre les générations successives de dieux, de Titans, de Géants et d'humains -, un psychopompe - celle qui ingère le sang et les restes de ces créatures lors de leur dernier voyage - et une régénératrice - celle qui réorganise et régénère ces restes pour engendrer les générations suivantes. Si bien que ce récit mythique semble entrer en résonnance avec la fin du monde annoncée par des scientifiques : "À l'image des Géants écrasés sous une pluie de pierre, les crises écologique, sociale et politique qui secouent notre monde contemporain seraient autant de symptômes de l'effondrement probable de notre civilisation et du déclin de nos sociétés." (Claeys \& Lambert, 2018)

Le sentiment partagé qu'une cause extérieure - transcendante ou naturelle - aura pour effet la fin du monde réapparaît régulièrement dans les sociétés humaines. Ainsi, à partir d'un extrait obscur de l'Apocalypse de saint Jean - écrite au I ${ }^{\text {er }}$ siècle avant J.-C. - annonçant le retour sur terre de Satan mille ans après l'incarnation du Christ, des érudits de la Renaissance auraient développés le mythe historique des peurs de l'an mille, bien avant les peurs irrationnelles du bug de l'an 2000. Encore aujourd'hui, de nombreux films hollywoodiens utilisent le thème de la catastrophe planétaire pour susciter dans l'esprit de leurs spectateurs une fascinante expérience cathartique (collision de météorite avec la terre, débarquement d'extra-terrestres, épuisement des ressources naturelles, virus mondialisé, 
zombies...). Tandis que les astrophysiciens affirment avec gravité que la mort du soleil devrait entraîner la fin de notre monde dans environ cinq milliards d'années.

La cause première de la crise multiforme actuelle serait d'abord l'inadéquation entre le mode de développement de l'espèce humaine et la rareté relative des ressources disponibles sur terre qui ne permettrait plus à l'humanité de subvenir à ses besoins.

L'être humain est "équipé initialement d'un inné insuffisant pour survivre naturellement et affecté d'une connaissance limitée du réel malgré l'acquis de ses expériences" (Claeys, 2017). Comme tout système vivant, il est d'abord "inachevé" parce qu'il a "des besoins physiologiques fondamentaux" dont "la satisfaction est nécessaire pour sa survie" et dont "la plupart nécessitent des actions dans le réel" (oxygène, eau, nourriture, température corporelle constante, élimination, logement, repos, reproduction). Mais lorsque ces besoins premiers sont assouvis, l'être humain est également "incomplet" parce qu'il a "des besoins psychologiques et sociaux fondamentaux", nécessaires pour contrebalancer l'angoisse existentielle liée à la coupure du réel opérée par le langage rythmant ses états de conscience.

Tout être humain devant survivre se comporte donc en tant qu'être auto-organisé négociant perpétuellement, à la fois, avec les affres de son propre état psychosomatique, avec les autres membres de la société à laquelle il appartient et avec les effets de l'environnement écosystémique dans lequel il évolue. S'il entretient inévitablement une relation intime avec sa psychè personnelle, il projette sur les autres et sur l'environnement des valeurs et des règles évolutives à partir desquelles ils entre en relations avec eux. Ce qui pose la question de l'existence de ce qui pourrait être appelé un contrat révisable, respectivement social et naturel. Or, au cours de l'histoire, la manière avec laquelle l'être humain a imaginé ce double contrat a modifié les chances de survie de son espèce.

Mais comment en sommes-nous arrivés à enviseager l'effondrement imminent de notre civilisation ?

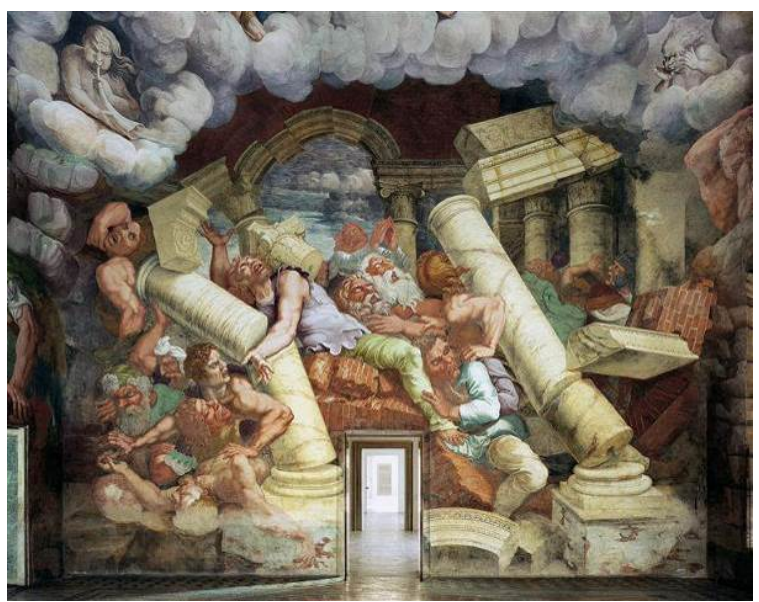

Fig.1 - Jules Romain, La Chute des Géants, Fresque du mur nord de la salle des Géants, palais du Té, Mantoue, Italie, 1532-1534.

\section{DU LA CROISSANCE À LA DÉCROISSANCE}

La reprise de conscience du rapport de dépendance des sociétés humaines à leur environnement a été très lente. De plus, l'observation de la nature a d'abord été menée de manière autoréférentielle, puisque l'être humain a commencé par étudier la nature... de l'être humain.

Jusqu'au XVIII ${ }^{\mathrm{e}}$ siècle, la nature est notamment personnifiée par la déesse égyptienne Isis (devenue plus tard la déesse grecque Artémis) sous les traits d'une jeune femme cachant sous un voile léger le secret de sa structure et de son fonctionnement. Aux XVII et XVIII ${ }^{\mathrm{e}}$ siècles, la science moderne est alors celle qui tenter de lever le voile par la ruse prométhéenne, tandis que les beaux-arts orphiques tentent de révéler ses secrets en épousant avec harmonie son rythme. 
À la fin du XVII ${ }^{\mathrm{e}}$ siècle, les fondateurs de libéralisme économique ayant mené au développement antagoniste des idéologies socialistes et capitalistes exprimaient déjà des craintes aux sujets des dérives possibles des théories économiques qu'ils inventaient du point de vue du rapport aux ressources planétaires et des rapports entre les membres de la société. Mais leurs héritiers contemporains - les tenants de l'idéologie du néocapitalisme - ont largement occulté cette partie de leur pensée.

Avant la première révolution industrielle, le libéralisme émergent propose la protection des droits fondamentaux naturels de chaque individu - menant à la future Déclaration des droits de l'homme et du citoyen (1789) - en régulant les interactions entre les êtres humains - le futur "contrat social" de Jean-Jacques Rousseau (1762) - et en mettant l'État au service de ses citoyens (l'émergence des démocraties parlementaires).

De manière radicalement utilitariste, dans le Discours de la méthode (1637), le philosophe français René Descartes écrit que la science peut "nous rendre comme maîtres et possesseurs de la nature". La nature est alors dominée pour répondre aux besoins humains. De manière un peu plus nuancée, dans le Deuxième Traité du gouvernement civil (1690), le philosophe anglais John Locke affirme que l'être humain possède un droit fondamental de propriété, mais il ajoute que ce droit est limité à ce qui est nécessaire à sa conservation. Selon lui, "Dieu nous a donné toutes choses en abondance", mais "Dieu n'a rien créé pour que l'homme le gaspille ou le détruise". Donc "Tout ce qu'un homme peut utiliser de manière à en retirer quelque avantage quelconque pour son existence sans gaspiller, voilà ce que son travail peut marquer du sceau de la propriété. Tout ce qui va au-delà excède sa part et appartient à d'autres". Il justifie son raisonnement en expliquant que "Quand Dieu a donné le monde en commun à toute l'humanité, il a enjoint à l"homme de travailler; d'ailleurs, l'homme s'y voyait contraint par la pénurie de sa condition", de là, "Dieu et la raison lui commandaient de venir à bout de la terre, c'est-àdire de l'améliorer dans l'intérêt de la vie" en s'investissant par son "travail". Autrement dit, l'individu a le droit de s'approprier les richesses naturelles par son travail - c'est même un devoir face à Dieu et à la raison - à condition que la part prélevée soit raisonnable de manière à en laisser pour les autres. En conclusion, tout être humain doit avoir accès aux ressources terrestres. Au XVIII ${ }^{\mathrm{e}}$ siècle, dans la Théorie des sentiments moraux (1759), le philosophe et économiste écossais des Lumières Adam Smith - souvent décrit de manière réductrice comme l'un des pères des sciences économiques modernes et du libéralisme économique - proposera même une philosophie morale à la recherche des causes de l'immédiateté et de l'universalité des jugements moraux chez l'être humain à partir d'un double processus de "sympathie".

À l'époque des Lumières, face aux inégalités sociales, aux développements des sciences modernes et de la première révolution industrielle, des penseurs considèrent la nature comme l'état originel de l'être humain. Dans Du contrat social (1762), le philosophe suisse Jean-Jacques Rousseau affirme que l'être humain vit au départ un "état de nature" où il est naturellement bon, compatissant et vertueux - la nature est ici associée à la beauté, l'innocence et la vertu -, mais que malheureusement la société le corrompt - la civilisation fait passer du bonheur à la misère. L'instauration de la vie en société est inévitable mais regrettable. Il propose alors la description d'une société différente dirigée non plus par la monarchie, l'aristocratie et l'Église, mais par tous les citoyens. Les révolutionnaires développeront par la suite ces idées en établissant la République. Mais seules les inégalités naturelles entre les êtres humains sont défendues pour mener à l'élaboration d'un contrat social. La nature n'est pas défendue pour elle-même, aucun contrat naturel n'existe tel que nous pourrions l'envisager aujourd'hui. Individu et société semblent s'accorder sur la nécessité d'élaborer un contrat social, alors que le contrat naturel semble, au mieux, implicite.

Avant la première révolution industrielle, l'abondance des ressources naturelles est l'expression de la bonté divine et il est impensable qu'elle puisse être menacée. Mais dès le milieu du XIX ${ }^{\mathrm{e}}$ siècle, l'idée émerge dans les consciences que nos ressources planétaires puissent disparaître. Ainsi, dans un élan romantique, les peintres de l'école de Barbizon habitués à peindre en plein air et d'après nature dans la forêt de Fontainebleau demandent dans un souci au départ esthétique la création de réserves artistiques. De même, le forestier américain Aldo Léopold (1949) - pionnier de l'écologie - crée une éthique environnementale menant à la création des réserves biologiques. 
Plutôt que de rejeter la responsabilité de toute fin du monde potentielle sur l'effet d'une hypothétique cause extérieure, les scientifiques prennent très progressivement conscience qu'une séparation totale entre nature et être humain est illusoire. Ils finissent par penser qu'une cause immanente au fonctionnement de l'espèce humaine puisse avoir pour effet un déséquilibre dans l'environnement, ce qui les amène à replacer progressivement l'espèce humaine en tant que simple élément parmi d'autres d'un écosystème dont la survie dépend de ses interactions avec les autres.

Après les ténèbres de la Seconde Guerre mondiale marquant la faillite du système socioéconomique hérité en grande partie des Lumières, les penseurs se sont demandés si l'espèce humaine était encore capable d'habiter le monde après une telle catastrophe. L'idée d'un possible effondrement planétaire prend racine dans le traumatisme d'Hiroshima menant à l'angoisse de la Guerre froide et à la vague contestataire de la fin des années 1960.

En raison d'effets négatifs contre l'humanité attribués au progrès, le concept de décroissance est apparu progressivement, au moins depuis les années 1970, lorsqu'il a été popularisé après la réunion et la traduction de textes de l'économiste d'origine roumaine Nicholas Georgescu-Roegen (qui n'emploie pas le mot) par Jacques Grinevald et Ivo Rens dans Demain la décroissance. Entropie, écologie, économie (1979). En introduisant les concepts d'entropie et d'évolution dans la théorie économique, il démontre qu'il est impossible de produire de manière infinie dans un monde fini. Alors que le mythe du progrès était jusque-là associé au développement de l'humanité, il semblait devenir synonyme de régression, de misère sociale et de catastrophe écologique.

La décroissance constitue - selon Pablo Servigne et Raphaël Stevens (2015) - un programme politique de "frugalité" et de "convivialité" destiné à éviter l'effondrement, proposant "une réduction graduelle, maîtrisée et volontaire de nos consommations matérielles et énergétiques". En partant de l'hypothèse que l'effondrement est maintenant inéluctable, ils pensent que ce programme "n'est pas très réaliste". Par contre, ils pensent que le concept d'effondrement "conserve la possibilité de penser un avenir qui ne soit pas totalement maîtrisé". Selon eux, les "euphémismes optimistes" annonçant l'émergence potentielle d'un monde meilleur tels que la "mutation" d'Albert Jacquard (2004), la "métamorphose" d'Edgar Morin (2011) ou la "transition" de Rob Hopkins (2008) sont utiles pour "ouvrir l'imaginaire d'un avenir pas forcément nihiliste ou apocalyptique", donner de l'espoir et rendre volontaire les foules, mais ils mettent de côté l'urgence de la situation.

\section{DES STRUCTURES STATIQUES AU SYSTÈMES DYNAMIQUES}

Alors que le biologiste d'origine autrichienne Karl Ludwig von Bertalanffy (1968) fonde la pensée systémique destinée à modéliser des systèmes ouverts - impossibles à modéliser à l'aide de théories déterministes et mécanistes -, le concept d'autonomie est devenu central dans l'étude des systèmes vivants, humains et sociaux à partir du moment où les systémiciens les ont étudiés en intégrant leurs capacités à s'auto-organiser. De là, Humberto R. Maturana \& Francisco J. Varela (1972) ont défini le concept d'auto-poïèse issu des deux mots grecs autos (soi) et poiein (produire). L' "auto-production" est donc l'aptitude et la propriété fondamentale de tout organisme vivant de s'auto-produire, c'est-àdire de produire sans cesse sa propre existence, et de s' "auto-réguler". Ils développent la théorie des systèmes "auto-poiétiques", à la fois, fermés et ouverts. En effet, ils sont fermés au niveau fonctionnel (ils maintiennent leur "identité" pour maintenir leur "clôture opérationnelle"), alors qu'ils restent ouverts au niveau structurel grâce à l'action constante des boucles de rétroaction qui les constituent (le système complexifie ses composants ou produit dans/par ses interactions avec son environnement de nouvelles configurations, il intègre de nouveaux composants depuis l'environnement ou il supprime certains de ses composants). Pour s' "auto-organiser", d'un côté, le système fait évoluer son "comportement" et, de l'autre, sa "structure". Un phénomène est donc modélisable non comme une structure statique résistante, mais comme un système dynamique résilient.

Lors de son émergence dans les années 1960, la pensée systémique est pionnière dans la prise de conscience de la limite des ressources disponibles sur terre. Dans Urban Dynamics (1969), Jay W. Forrester a développé le modèle World2 inspiré de la théorie générale des systèmes pour - comme l'écrit Pierre-Henri Derycke dans la préface à l'édition française de 1978 - étudier les "conséquences 
lointaines des décisions de politique urbaine" et explorer l'avenir des "cités géantes", avec une méthode très différentes des multiples "modèles explicatifs généraux de la croissance urbaine" des années 1960 autour de la Rand Corporation. Très critiqué selon ses contradicteurs, à la fois, pour son simplisme ou pour sa complexité, le modèle de Forrester est constitué de trois sous-systèmes articulés entre eux. Au sein de ces sous-systèmes, les distinctions opérées entre les sous-classes internes paraissent douteuses et politiquement incorrectes aujourd'hui, mais elles doivent être replacées dans le contexte de l'époque. Selon Forrester, le modèle traite du "processus de croissance des aires urbaines" et de l'étude de leur "cycle de vie". Une "aire urbaine" est "un système formé d'industries, de logements et de personnes en interrelations" :

1. les entreprises (ou activités économiques) parcourant un cycle vital: de jeunes à mûres jusqu'au déclin ;

2. les logements divisés en trois classes occupées par trois classes sociales étanches: les logements de standing, les logements de confort, les logements déclassés et les taudis ;

3. la population active divisées en trois classes : les dirigeants et les managers, les travailleurs qualifiés, les travailleurs sans qualification (avec les chômeurs et les marginaux, les immigrés et les femmes !).

Ces trois sous-systèmes sont articulés entre eux à partir de règles. Par exemple, les logements de standing sont moins densifiés que les logements ordinaires, à leur tour moins peuplés que les logements déclassés, tandis que la taille moyenne des familles augmente en fonction du degré de paupérisation. Des transformations du système sont introduites dans le modèle par des impulsions venues de l'extérieur et la régulation est opérée par des variables d'état (des stocks) et des mécanismes de rétroaction (des boucles) pour ramener le système à l'équilibre. Les projections ont été élaborées sur 250 ans ! Ce qui est l'une des critiques habituelles du modèle.

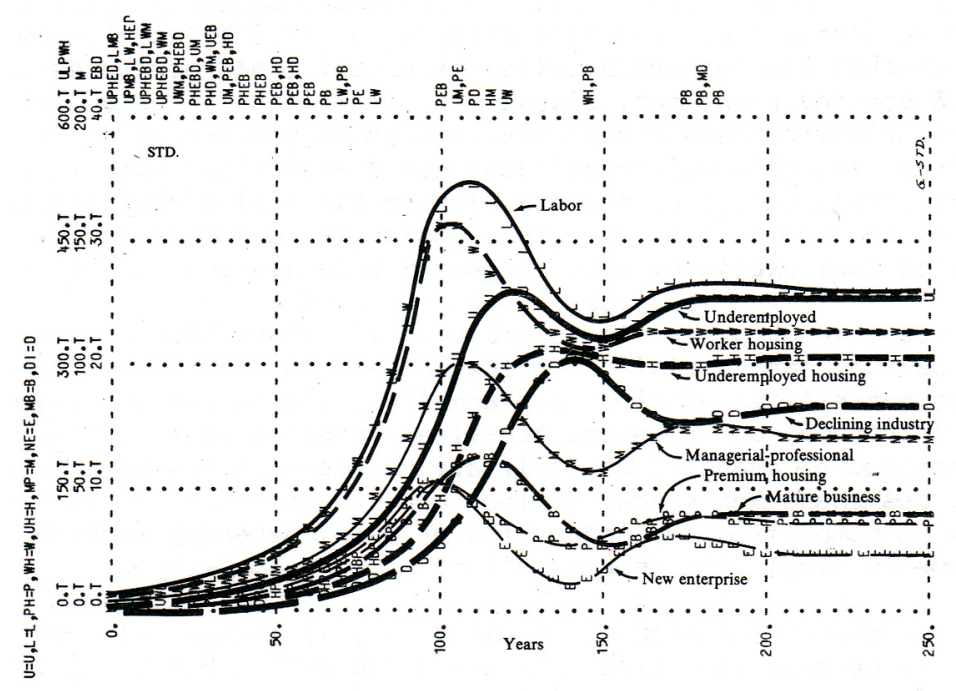

Fig.2 - Exemple de cycle de vie d'une aire urbaine : 250 ans de développement interne, de maturité et de stagnation (Forrester, 1969, p.4).

Plus tard, les travaux de Forrester se prolongent dans l'historique et controversé Rapport Meadows, diffusé dans The Limits to Growth: A report for The Club of Rome's project on the predicament of mankind (Meadows, Meadows, Randers, Behrens III, 1972), traduit en français par Halte à la croissance?, avant d'être réactualisé récemment dans The Limits to Growth: the 30-Year Update (Meadows, Meadows, Randers, 2004) ${ }^{1}$. Ainsi, dans Limits to Growth, les auteurs publient l'analyse

1 L'américain Jay W. Forrester est un théoricien de la dynamique des systèmes et un pionnier en modélisation informatique. Après avoir travaillé notamment sur Urban Dynamics (1969), il tente une réponse aux questions soulevées par le Club de Rome - un groupe informel et international d'hommes d'affaires, de dirigeants et de scientifiques - sur les équilibres 
prospective de douze scénarios à partir de la modélisation dynamique des systèmes basée sur le modèle World3 de Jay W. Forrester en montrant "différents modes de développement de l'humanité sur deux siècles", entre 1900 et 2100. À cette époque, les débuts de l'informatique fournissent de nouveaux outils de calcul et de modélisation pour étudier le monde en tant que système terre évolutif. Ce modèle tient compte de différentes dimensions environnementales, sociétales et économiques et tente de montrer leurs interactions entre les principaux paramètres globaux du monde qu'ils identifient: la population, la production industrielle, la production des services, la production alimentaire, le niveau de pollution et les ressources non-renouvelables. Le rapport remettait en cause le mythe de la croissance sans fin. Il avait alerté l'humanité sur les conséquences à long terme d'une économie fondée sur le principe de la croissance exponentielle - illustré par des boucles de rétroactions positives - sans que rien ne vienne contrebalancer ce mouvement global. La conclusion principale du rapport tient en une phrase : en partant de l'hypothèse que notre monde possède des limites physiques, "un effondrement généralisé de notre civilisation thermo-industrielle aura très probablement lieu durant la première moitié du XXI siècle" (Servigne \& Stevens, 2015). Les questions traitées par ce groupe dans les années 1970 étaient par exemple: "les politiques actuelles nous conduisent-elles vers un avenir soutenable ou vers l'effondrement? Que peut-on faire pour créer une économie humaine qui fournisse de tout en quantité suffisante à tous ?". Il ne revendiquait pas encore une décroissance, mais une "croissance zéro". Autrement dit, que toutes les activités économiques tendent vers un état stable. L'année suivant la publication du Rapport Meadows, la crise pétrolière de 1973 fera prendre conscience du problème de l'épuisement des ressources au public. À l'intérieur du modèle World3, des seuils ont pu être définis, mais ce type de seuil est trop complexe à déterminer dans le réel.

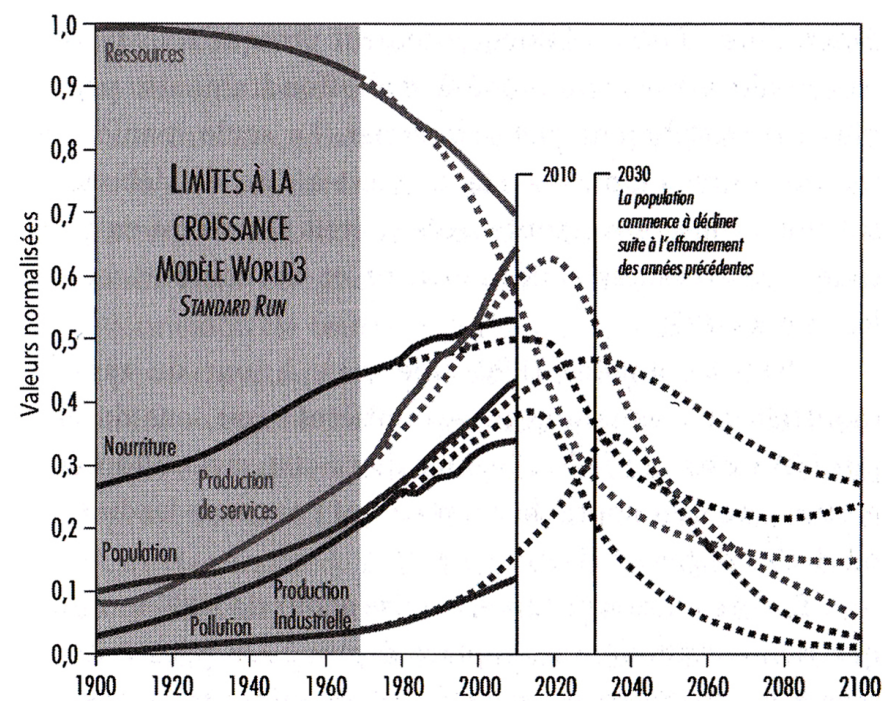

Fig.3 - Modèle "Standard Run" (Rapport Meadows, 1972), mis à jour par Graham M. Turner (2012) et repris par Pablo Servigne \& Raphaël Stevens (2015).

Selon ces chercheurs, tous les scénarios alternatifs testés avec le modèle World3 mènent à l'effondrement de la société, la seule possibilité de rendre notre monde "stable" pour arriver à une civilisation "soutenable" est de mettre en place toutes ces mesures simultanément dès les années 1980.

globaux en ébauchant un modèle dynamique de base : le World1. Ensuite, il documente le modèle final - World 2 - dans Worlds Dynamics (1971) qui traite de la modélisation des interactions complexes dans les sphères économique, démographique et environnementale. Enfin, en développant la structure et en enrichissant quantitativement la base de données, il confie le travail de développement d'un troisième modèle - le World3 - à Donella H. et Denis L. Meadows au sein du Groupe Dynamique des Systèmes de la Sloan School of Management du Massachusetts Institute of Technology (MIT) entre 1970 et 1972. Ces modélisations servent de matrice au Rapport Meadows (1972) qui prévoyait un effondrement dans 50 ou 100 ans en cas de croissance exponentielle non maîtrisée des sociétés humaines et de leur consommation. 
Une mise à jour du rapport dans les années 1990 montrait que les limites existaient et que notre civilisation s'approchait de certaines limites et dépassait même certaines frontières. Dans une autre mise à jour du rapport en 2004, les auteurs ont montré que rien n'avait été mis en place pour éviter le scénario "business as usual" depuis 1972 et qu'il restait une "toute petite fenêtre d'opportunité à ne pas manquer" pour éviter un effondrement global. Ce rapport montre l'extrême instabilité du système qui gère notre civilisation, l'interaction entre les différentes crises qui l'agitent et l'intérêt d'une pensée systémique.

Plus récemment, le chercheur australien Graham M. Turner (2012) a comparé les résultats de la simulation World 3 avec les données réelles en 2008 et en 2012 : il affirme que le scénario du Rapport Meadows qui se rapproche le plus du réel est le pire scénario : le "standard run" ["modèle standard"] ou scénario "business as usual".

\section{DE LA RESPONSABILITÉ À LA SOUTENABILITÉ}

Modéliser un système vivant - physique, social ou humain - en le dotant d'une autonomie ne signifie pas qu'une liberté totale d'action lui est attribuée. Il est conçu en interaction étroite avec le contexte dans lequel il évolue et des scénarios sont définis pour prévoir les conséquences futures de ses réactions présentes. Autrement dit, si les valeurs des variables à partir desquelles des simulations sont lancées dépassent des seuils critiques, le système peut altérer son fonctionnement futur. La question de l'autonomie mène à celle de la responsabilité.

À partir d'études de systèmes institutionnalisés dans des domaines différents (école, médecine, transports, énergie...), Ivan Illich développe dans ses écrits une théorie de la contre-productivité liée au concept de seuil. Selon lui, "lorsque l'institution se développe et dépasse une certaine limite ou un certain seuil, elle devient nuisible et fortement dysfonctionnelle par rapport à ses propres objectifs et finalités. L'institution devient alors contre-production, contre-productivité. Elle aliène l'être humain et détruit la société globale" (Assogha, 1979). Autrement dit: "Lorsqu'une activité outillée dépasse un seuil défini par l'échelle ad hoc, elle se retourne d'abord contre sa fin, puis menace de destruction le corps social tout entier." (Illich, 1973). Parallèlement aux travaux de plusieurs systémiciens, Illich montre que des seuils identifiables sont capables de faire basculer tout système institutionnalisé de l'autonomie vers l'hétéronomie et la dépendance. Dans la société moderne, il associe ces seuils à un déséquilibre entre l'usage d'outils conviviaux (maniables, accessibles à tous) et celui d'outils manipulables qui reposent sur des technologies maîtrisée par des spécialistes et dont la fabrication relève d'un processus industriel. C'est ainsi qu'il préconise un débat public sur l'établissement de seuils. Selon Illich, l'autonomie est d'abord rendue possible par l'acceptation et le choix de limites autoimposées à soi-même ou au système.

Dans Le principe responsabilité (1979), Hans Jonas part du constat que "Le Prométhée définitivement déchaîné, auquel la science confère des forces encore jamais connues et l'économie son impulsion effrénée, réclame une éthique qui, par des entraves librement consenties, empêche le pouvoir de l'homme de devenir une malédiction pour lui". Il affirme que l'espèce a le pouvoir technologique de s'autodétruire et ce pouvoir est aux mains de quelques individus ayant la responsabilité de l'humanité entière. Parallèlement à un pouvoir plus grand, de nouveaux devoirs apparaissent pour l'être humain. Selon lui, l'éthique traditionnelle reposait sur l'évaluation des actions (par exemple, mentir, c'est mal), alors qu'aujourd'hui elle devrait porter sur l'évaluation de la conséquence des actions (par exemple, moins consommer est bien pour anticiper l'épuisement futur des ressources planétaires). Le concept de responsabilité résume ce devoir moral nouvellement construit par l'intégration de la question de l'avenir (la conséquence des actes) et de la planète entière (la conséquence sur l'écosystème global de ces mêmes actes). Il va jusqu'à écrire que les êtres humains doivent questionner l' "impératif inconditionnel de préserver leur existence pour l'avenir" en prenant en compte "la vulnérabilité critique de la nature par l'intervention technique de l'homme". L'être humain doit donc adapter son projet téléologique pour qu'il réponde à des principes éthiques traditionnels, mais également à d'autres qui ne sont plus uniquement anthropocentriques à partir du moment où ils intègrent la phúsis. À une plus grande autonomie de l'être humain grâce à la technologie correspondrait donc une plus grande responsabilité. Pour Hans Jonas, l'être humain a d'abord l'obligation directe de solidarité 
intergénérationnelle pour éviter que les actions présentes entravent les possibilités futures de la vie humaine sur terre. Cette première obligation en entraîne une seconde, l'obligation indirecte à l'égard de la nature contribuant à la préservation des conditions d'existence de l'humanité et pour sa valeur intrinsèque indépendante de tout usage. Cette double obligation mène au concept de développement durable.

À l'époque, les chercheurs reprennent conscience que la nature doit être préservée parce qu'elle conditionne l'existence même de l'humanité. La responsabilité n'est plus uniquement celle du présent, mais elle est également celle du futur intergénérationnel. En 1983, l'Organisation des Nations Unies crée une Commission mondiale sur l'environnement et le développement pour définir un programme pluridisciplinaire de coopération internationale pour étudier les problèmes environnementaux. Présidée par la première ministre norvégienne Gro Harlem Brundtland, la commission a rédigé Our Common Future [Notre avenir à tous] (1987) - dit le Rapport Brundtland - qui servira de programme de base au Sommet de la Terre de 1992 et qui définit le concept de sustainable development [développement durable] comme "un mode de développement qui répond aux besoins des générations présentes sans compromettre la capacité des générations futures de répondre aux leurs " ${ }^{2}$. Il ajoute que deux concepts sont inhérents à cette notion : le concept de besoins, et plus particulièrement des besoins essentiels des plus démunis, à qui il convient d'accorder la plus grande priorité, et l'idée des limitations que l'état de nos techniques et de notre organisation sociale impose sur la capacité de l'environnement à répondre aux besoins actuels et à venir". Deux préoccupations reviennent encore : les besoins fondamentaux de l'être humain et les limites des ressources disponibles.
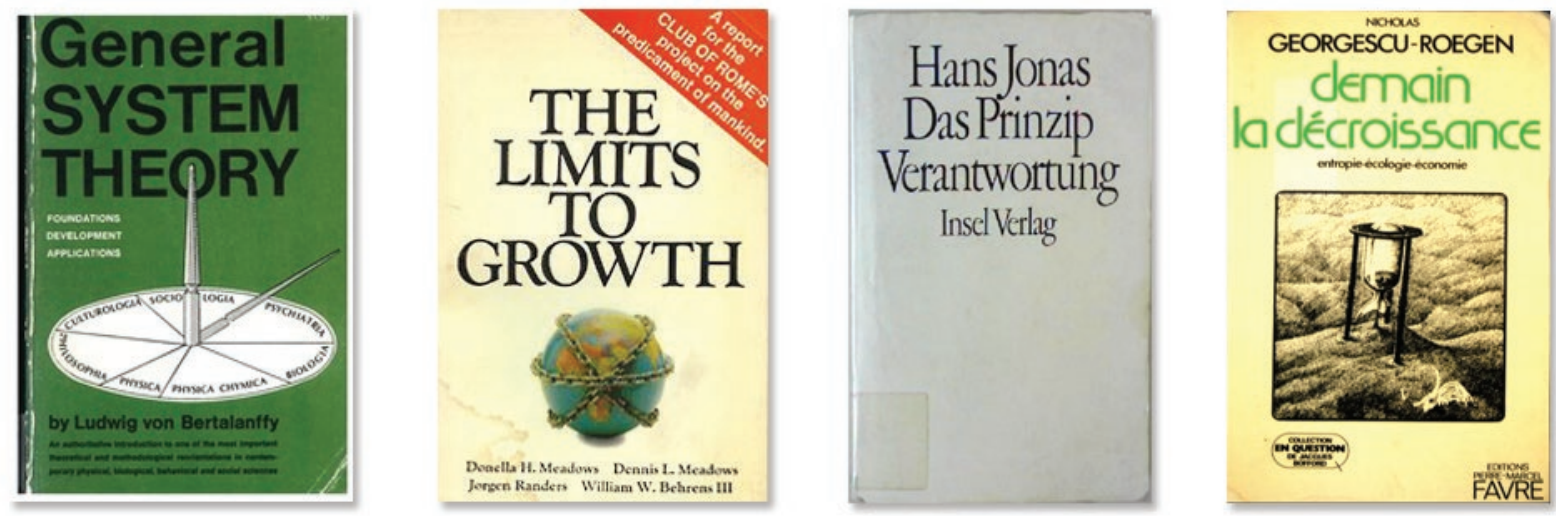

Fig.4 - Premières de couvertures emblématiques

(Bertalanffy, 1968 ; Rapport Meadows, 1972 ; Jonas, 1973 ; Georgescu-Roegan, 1979).

\section{DE L'ANTHROPOCÈNE À LA CAPITALOCÈNE}

Plus récemment, des scientifiques tentent de définir une nouvelle ère géologique - l'anthropocène - et une nouvelle science - la collapsologie - pour étudier les causes et les effets de l'effondrement potentiel de notre civilisation.

Composé des mots grecs anthropos (être humain) et kainos (récent, nouveau), le mot anthropocène l'âge de l'homme - possède une origine pluridisciplinaire difficile à retracer (Hamilton \& Grinevald, 2015 ; Lewis \& Maslin, 2015). L'anthropocène désigne une période géologique caractérisant l'époque terrestre ayant débuté lorsque les activités humaines ont eu un impact global significatif sur l'écosystème terrestre. Ce constat n'est pas neuf puisque dans l'un des premiers ouvrages sur l'écologie, Man and Nature (1864), réédité sous le titre The Earth as Modified by Human Action (1885), l'américain George P. Marsh montre que la déforestation mène à la désertification et que "l'action de

\footnotetext{
${ }^{2}$ Le développement durable repose sur trois piliers : écologique, social et économique. L'un de ses trois piliers étant l'économie, l'expression est controversé puisqu'elle semble fortement liée à l'idée de croissance économique. Le mot soutenabilité qui vient du mot anglais sustainability, lui-même issu du latin sustinere (soutenir, empêcher de tomber, porter, supporter) lui est parfois préféré.
} 
l'homme pourrait conduire à un environnement aussi désolé que celui de la lune". Par ailleurs, selon lui, le géologue italien Antonio Stoppani (1873) aurait imaginé dans son cours de géologie une ère géologique nouvelle appelée l' "êra antropozoica" [l'ère anthropozoïque]. Alors qu'il était également théologien, Stoppani énonce que : "La création de l'homme a été l'introduction d'un nouvel élément dans la nature, d'une force totalement inconnue des périodes précédentes". Il compare l'espèce humaine à "une nouvelle force tellurique qui, en puissance et en universalité, peut être comparée aux plus grandes forces de la terre". Citons encore le géologue russe Aleksei Pavlov qui aurait décrit les temps actuels comme ceux d'une période "anthropogène" en 1925.

Durant les années 1980, le biologiste américain Eugene F. Stoermer utilise le concept d'anthropocène dans un sens complètement différent de limitation nécessaire de l'évolution. De manière prophétique, le journaliste américain Andrew Revkin (1992) avait écrit: "Peut-être que les géologues de l'avenir nommeront cette nouvelle période post-holocène pour son élément causal - pour nous. Nous entrons dans une ère que l'on pourrait appeler un jour, disons, l'Anthrocène. Après tout, c'est un âge géologique que nous avons nous-mêmes créé". Finalement, il reviendrait au météorologue et chimiste de l'atmosphère néerlandais Paul J. Crutzen (2002) - menant des recherches sur le trou de la couche d'ozone et ayant travaillé avec Stoermer (Crutzen \& Stoermer, 2000) - d'avoir popularisé le concept d'anthropocène tel que nous le connaissons aujourd'hui.

L'anthropocène est "à la fois une période de domination du monde par l'espèce humaine, mais également le signe d'une fin imminente de celle-ci" (Claeys, 2017). Ainsi, le sociologue français Edgar Morin (1973) montre que l' "homo demens", avec son comportement irresponsable, porte en lui les germes de sa propre extinction. D'ailleurs, à force de vivre à crédit ${ }^{3}$, le scientifique australien Frank J. Fenner (2010) prédit l'extinction de l'espèce humaine "dans les 100 années qui viennent". Tout comme dans le mythe, Gaïa - la terre - est, à la fois, la mère de toutes les générations successives de dieux et d'êtres humains et celle qui absorbe leurs restes sans aucun état d'âme. Ainsi, le professeur anglais de paléobiologie Michael Boulter (2002) affirme que la nature est un système auto-organisé capable de muter lorsqu'un déséquilibre apparait. Selon lui, la planète s'adaptera et survivra, mais l'humanité probablement pas! Tout simplement parce que, comme l'a dit le prix Nobel belge de médecine Christian de Duve : "La nature est indifférente !"

À partir du moment où la Terre est considérée comme un organisme global et auto-organisé à la recherche d'un équilibre indépendamment de l'action humaine, les effets du contrat social semblent bien insignifiants par rapport à ceux du contrat naturel. Si bien que cautionner une organisation politique et économique détruisant l'environnement semble désuet et irresponsable. En effet, en reprenant l'expression de l'historien britannique Eric J. Hobsbawm (1975), l'écologue suédois Andreas Malm (2016), spécialisé en "écologie humaine", affirme que nous ne vivrions pas à l' "âge de l'homme" mais bien à l' "'âge du capital". Plutôt que de parler d'anthropocène, il serait donc plus juste de parler de "capitalocène". Il suggère que ce ne serait pas l'activité humaine en soi qui menacerait de détruire notre planète, mais bien l'activité humaine telle que mise en forme par le mode de production capitaliste.

Alors que le contrat naturel était pensé à travers le prisme du contrat social, cette fois le second est actualisé dans une version planétaire à partir des concepts de biosphère et de noosphère. Au départ, le géologue autrichien Eduard Suess utilise pour la première fois le concept de "biosphère" en 1875, ce qui permettra au minéralogiste russe Vladimir I. Vernadsky (1926) d'en définir les aspects biogéologique et écologique, avant que le botaniste britannique Arthur G. Tansley (1935) ne développe le concept d' "écosystème". Plus tard, dans un glissement des écosystèmes aux systèmes

3 Chaque année l'Institut international de recherche Global Footprint Network calcule le controversé "Earth Overshoot Day" $(E O D)$ ou en français "jour du dépassement" correspondant au jour de l'année à partir duquel l'humanité aurait consommé la totalité des ressources que la terre serait capable de régénérer en un an. À partir de cette date, l'humanité vit à crédit pour le reste de l'année. Pour le calcul : $E O D=$ (biocapacité de la planète / empreinte écologique de l'humanité) x 365 . L'EOD aurait été atteint le 29 décembre en 1970, le 3 novembre en 1980, le 11 octobre en 1990, le 23 septembre en 2000, le 8 août en 2010, le 1 août en 2018). Cette année, l'humanité consommerait donc 1,7 planètes Terre.

4 Christian de Duve s'exprima en ces mots lors de sa conférence d'ouverture du colloque international Darwinismes et spécificité de l'humain, intitulée "À l'écoute du vivant", le 28 avril 2009, à Louvain-la-Neuve. 
écosociaux, Vladimir I. Vernadsky (1945) introduira le concept de "noosphère" - le "monde de la pensée" ou la "sphère de la pensée humaine" - pour marquer le rôle croissant du cerveau humain dans le façonnement de son propre avenir et de son environnement, l'humanité devient une force géologique. Le concept sera notamment repris par le philosophe français Pierre Teilhard de Chardin (1955) et par Edgar Morin (2001). Pour Vernadsky et Teilhard de Chardin, ces concepts sont encore associés à une nécessaire évolution de l'humanité sous-tendue par le mythe du progrès, alors que chez Morin la noosphère permettrait le renouvellement d'un humanisme solidaire.

Bien que Simon L. Lewis et Mark A. Maslin (2015) aient fixé le début de l'ère anthropocénique à l'année 1610, la première révolution industrielle de la fin $\mathrm{du} \mathrm{XVIII}^{\mathrm{e}}$ siècle est souvent pointée comme l'une des causes principales ayant déclenché ce processus. Par ailleurs, une étape significative du phénomène correspondrait à l'année 1950 marquant le début de la grande accélération. Après la Seconde Guerre mondiale, cette période est marquée par la mondialisation de l'économie, par l'industrialisation planétaire, par les progrès technoscientifiques, par la course aux armements nucléaires, par l'explosion démographique, par la croissance rapide de l'économie et des inégalités...

Dans le sillage du projet synthétique de compréhension interdisciplinaire du Global Change affectant l'Earth system (la terre modélisée en système global et dynamique) mené par l'International Geosphere-Biosphere Program (IGBP) entre 1987 et 2015, des recherches montrent - pour différents indicateurs - des graphiques dans lesquels la grande accélération est clairement observable parce que des courbes possèdent une croissance exponentielle et de nombreux seuils critiques sont dépassés. Ces graphiques ont été mis à jour à plusieurs reprises (Steffen, Sanderson, Tyson et al., 2004 ; Steffen et al., 2015b). Or, dans tout monde fini, une société en croissance est confrontée à une biocapacité limitée et si elle dépasse ce seuil, elle s'effondre brutalement. Les courbes exponentielles de ces graphiques jouent avec les limites.

Après ces nombreuses recherches pluridisciplinaires - la définition d'une limite terrestre à la croissance, l'établissement de seuils critiques, l'adoption d'une nouvelle ère géologique et l'adoption d'une posture éthique durable - une nouvelle conception du monde a émergé : la terre est devenue un système global, complexe et évolutif, possédant une biocapacité limitée. De là, le développement actuel de l'humanité mènerait à l'effondrement à court terme du monde et à la disparition probable des sociétés humaines telles quelles sont organisées actuellement, voire même à celle de l'espèce humaine.
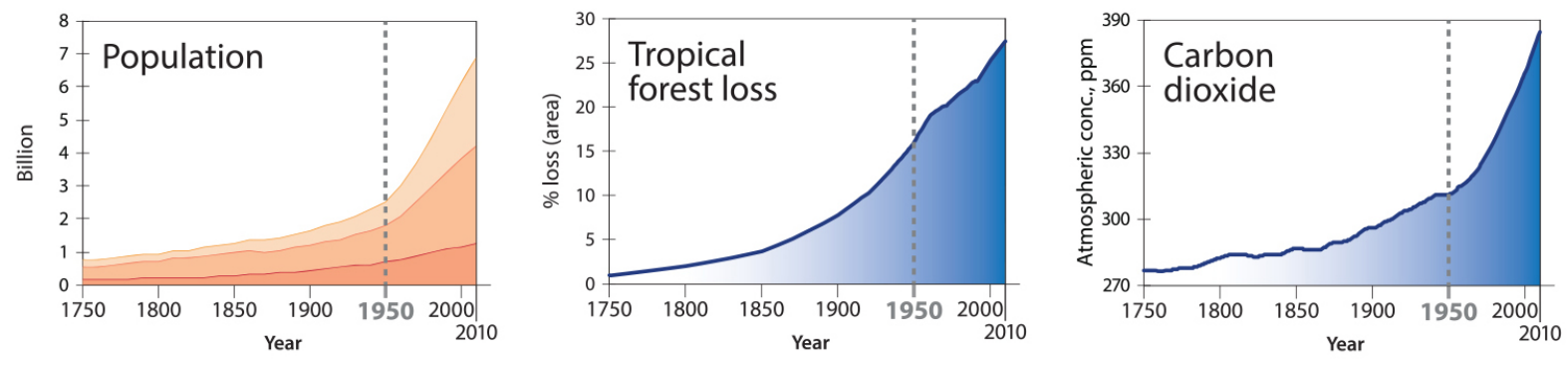

Fig.5 - Tendances entre 1750 et 2010 pour trois indicateurs parmi d'autres (Steffen et al., 2005a).

\section{DE L'HISTOIRE GLOBALE À LA COLLAPSOLOGIE}

Dans les années 1980, la Global History se développe et les chercheurs ont mis en évidence différentes causes à l'œuvre et leurs enchaînements lors de l'effondrement des civilisations, anciennes ou actuelles, en espérant modéliser et gérer ce type d'événement. Certains tentent de sauver nos systèmes écosociaux avec l'espoir de prolonger leur fonctionnement actuel alors que d'autres tentent de tirer parti de ces processus d'effondrement pour les modifier en profondeur.

Après l'analyse archéologique et historique de l'effondrement de civilisations anciennes, le géographe américain Jared Diamond (2005) étudie plutôt les limites physiques et écologiques du monde. Il définit l'effondrement, à partir des effets qu'il produit, comme "une réduction drastique de la population humaine et/ou de la complexité politique/économique/sociale, sur une zone étendue et une 
durée importante". Selon lui, l'effondrement est "une forme extrême de plusieurs types de déclins moindres". Il identifie cinq facteurs d'effondrement régulièrement à l'œuvre en synergie :

1. les dégradations environnementales et la pénurie de ressources, c'est-à-dire une exploitation des ressources au-delà de leur capacité à se reconstituer ;

2. le changement climatique ;

3. les guerres ;

4. la perte impromptue de partenaires commerciaux;

5. les mauvaises réactions de la société face au changement.

Selon lui, le facteur le plus puissant est écologique, mais le seul facteur commun a tous les effondrements est celui des dysfonctionnements internes de la société et de l'incapacité de celle-ci à prendre des décisions appropriées.

Dans The Five Stages of Collapse: Survivors' Toolkit (2013), l'ingénieur d'origine russe Dmitri Orlov a étudié l'effondrement de l'Union soviétique en le comparant à l'effondrement - selon lui - inévitable des États-Unis et il a défini cinq stades dans le processus d'effondrement par ordre de gravité croissant (les cinq étapes ne sont pas nécessaires pour qu'une société s'effondre) :

1. l'effondrement financier: les institutions financières deviennent insolvables et les banques ferment ;

2. l'effondrement économique: pénuries de biens essentiels et décomplexification de l'économie ;

3. l'effondrement politique : la corruption remplace les services de l'administration ;

4. l'effondrement social : perte des institutions sociales locales et guerre civile ;

5. l'effondrement culturel : perte de l'empathie et de l'humanité.

Un sixième stade a été ajouté plus tard :

6. l'effondrement écologique : redémarrer une société dans un écosystème épuisé est presque impossible.

Ensuite, d'autres chercheurs ont tenté de modéliser les processus d'effondrement dans les sociétés et dans les écosystèmes, entre autres, à partir de la théorie de la complexité et de la physique statistique.

Lorsque le cinéaste américain Godfrey Reggio initie sa trilogie des Qatsi - mise en musique par le compositeur américain Philip Glass -, le film Koyaanisqatsi (Reggio, 1982) popularise le genre du documentaire écologique - bien avant le succès de Demain (Dion \& Laurent, 2015) - et il montre la perte de contrôle de notre civilisation face au processus de croissance et de complexification qu'elle génère pourtant elle-même. À cette époque, dans The Collapse of Complex Societies (1988), l'anthropologue américain Joseph A. Tainter étudie l'effondrement de plusieurs sociétés anciennes à l'aide de la théorie de la complexité avec le prisme des limites socio-économiques. Il établit quatre concepts majeurs pour comprendre les effondrements, les trois premiers précédant le quatrième :

1. les sociétés humaines sont des organisations faites pour résoudre de problèmes (environnement, ennemis...);

2. les systèmes sociopolitiques ont besoin d'énergie pour se maintenir ;

3. la complexité accrue s'accompagne de coûts accrus par habitant ;

4. l'investissement dans la complexité sociopolitique en tant que réponse à la résolution de problèmes atteint souvent un point de rendements marginaux décroissants.

Selon lui, une société dont la complexité augmente peut être modélisée comme un système, "une société croît en complexité comme un système". Il définit que le dysfonctionnement politique d'une société menant à l'effondrement augmente avec la complexité croissante des institutions sociopolitiques qui augmentent leurs besoins en énergie et en matière. Des cycles d'essors et de déclins structurellement semblables semblent être observables dans l'histoire de l'humanité : le niveau de complexité d'une société augmente exponentiellement, un effondrement se produit, suivi d'une période de simplification institutionnelle... Il réalise un graphique qui présente la proposition suivante : "à un 
moment donné dans l'évolution d'une société, un investissement continu dans la complexité comme stratégie de résolution de problèmes produit un rendement marginal décroissant" ${ }^{5}$. Dans la figure, chaque fois qu'une société est soumise à un stress, elle investit dans sa propre complexification (augmentation des investissements dans l'agriculture ou dans d'autres ressources de production, dans la hiérarchisation, dans les processus d'information, dans l'éducation, dans la défense...). La courbe des "cost-benefit" (la courbe de rentabilité) est d'abord ascendante jusqu'à devenir contreproductive. À partir de ce point au sommet de la courbe, la société devient vulnérable à un effondrement potentiel. À mesure que le rendement marginal de l'investissement dans la complexité décline, la société investit toujours plus lourdement dans une stratégie proportionnellement moins rentable. Il faut alors faire face aux poussées de tensions en dehors du budget de fonctionnement courant. La complexité devient une stratégie globalisante de moins en moins séduisante, si bien que des parties d'une société perçoivent un avantage croissant à une politique de séparation ou de désintégration. Logiquement, divers segments de la population accroissent leur résistance active ou passive, ou tentent ouvertement de faire sécession. Selon la thèse de Tainter, l'investissement dans la complexité sociopolitique atteint un point où les bénéfices d'un tel investissement commencent à décliner, d'abord lentement, puis beaucoup plus rapidement. "Les sociétés s'écroulent par inertie, elles se montrent incapables de modifier leurs rapports à l'environnement quand celui-ci est altéré" (Testot, 2017).

Dans son modèle, Tainter affirme que ces cycles d'augmentation/diminution de la complexité sont réguliers dans l'histoire: l'étude de l'évolution des systèmes écosociaux devient progressivement cyclique.

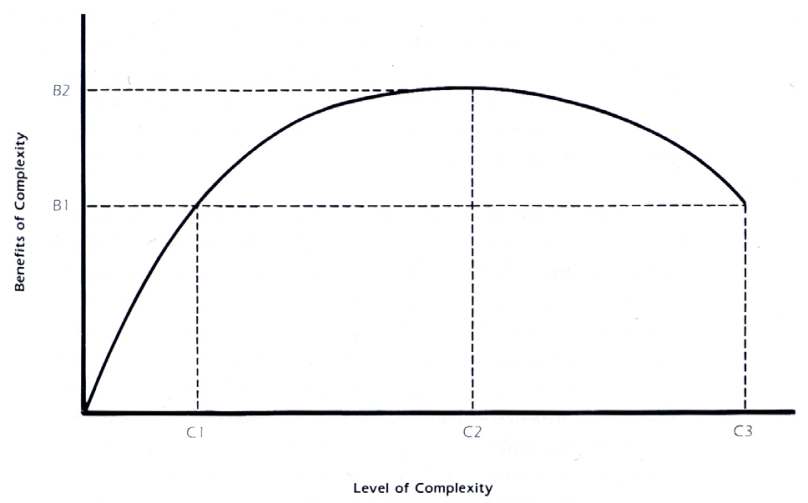

Fig.6 - The marginal product if increasing complexity (Tainter, 1988, Fig.19, p.119).

Plus récemment, le mathématicien Safa Motesharrei, le sociologue Jorge Rivas et la météorologiste argentine Eugenia Kalnay (2014) ont développé le modèle Human and Nature DYnamics (HANDY). Ce modèle est également controversé, notamment parce que ses contradicteurs le trouvent trop simpliste, sachant qu'il ne repose que sur quatre équations. Pourtant, selon ces chercheurs, HANDY reproduirait des comportements assez complexes avec une structure de modèle très simple, comparée à celle de modèles mondiaux tels que World3. Le modèle montre, en accord avec les "données historiques", que "la stratification économique ou la pression écologique peuvent conduire indépendamment à un effondrement". Il est construit sur un système d'équations proposé indépendamment par deux chercheurs dans les années 1920 - le mathématicien américain Alfred J. Lokta et le mathématicien italien Vito Volterra - pour décrire au départ une dynamique de compétition

\footnotetext{
5 En économie, la productivité marginale est la variation de la production de biens ou de services engendrée consécutivement à l'augmentation des moyens humains (travail, services), financiers (capital) ou de production (outils, machines). Le rendement marginal est donc la valeur de la variation de production obtenue divisée par le coût des moyens alloués pour l'obtenir. De là, la loi controversée des rendements décroissants affirme qu'après une phase de rendement croissant, l'augmentation du volume de production finira par mener à la diminution de la productivité marginale, alors le rendement décroitra progressivement.
} 
entre deux espèces, les interactions entre les populations de prédateurs et de proies en écologie ${ }^{6}$. Mais les êtres humains se distinguent des animaux par leurs capacités à s'organiser en sociétés, à utiliser des outils et à produire des stocks d'énergie, de matière, d'information. Contrairement aux animaux, ils ne subissent pas directement un déclin de population lorsqu'une ressource naturelle est épuisée. Ces recherches ont été transposées dans $H A N D Y$ pour le rendre capable de simuler dynamiquement la croissance démographique d'une société fictive en fonction de contraintes biophysiques: les prédateurs sont les êtres humains, tandis que la proie est son environnement. Les quatre équations du modèle décrivent l'évolution :

1. des "elites" (les élites), une petite partie de la population entre naissance et décès ;

2. des "commoners" (les citoyens), une plus grande partie de la population entre naissance et décès (la distinction originale de $H A N D Y$ entre élites et citoyens permet l'intégration de l'inégalité économique des membres d'une société.);

3. de la "nature" (la nature), les ressources de l'environnement entre régénération et épuisement ;

4. de la "wealth" (la richesse) de la société déterminée par le "surplus accumulé" ou la quantité globale de richesse accumulée (matière, énergie, information), déterminée en termes de consommation, entre production et épuisement.

Ils ajoutent la mesure de la "carrying capacity" (la charge admissible), c'est-à-dire "le niveau de la population que les ressources d'un environnement particulier peuvent soutenir sur le long terme", dont l'estimation est un moyen pratique pour détecter les effondrements.

Deux caractéristiques distinctes apparaissant dans toutes les sociétés qui se sont effondrées dans l'histoire sont modélisées :

1. "l'érosion des ressources en raison de la pression exercée sur la charge admissible" ;

2. "la stratification économique de la société" entre les élites et les citoyens.

Sur base de leur modèle, ces chercheurs montrent trois scénarios : (1) société égalitaire (tout le monde travaille et les richesses sont partagées entre tous), (2) société équitable (tout le monde ne travaille pas, mais les richesses sont partagées entre tous), (3) société inégalitaire (tout le monde ne travaille pas et les richesses ne sont pas partagées avec tous).

Le scénario le plus proche de notre situation contemporaine est celui d'une société inégalitaire qui épuise la nature ( $c f$. figure 7). Dans ce cas, un épuisement de la nature provoque une baisse de richesse, suivie d'une baisse de la population des citoyens et, enfin, des élites. Ce type de scénario caractérisé par "une trop grande exploitation des ressources" et "de trop grandes inégalités" mène à un type d'effondrement complet pouvant être irréversible. Selon eux, "une forte stratification sociale rend difficilement évitable un effondrement de civilisation" et, pour éviter cette issue, il faudrait: (1) réduire les inégalités économiques ; (2) maintenir la démographie en-dessous d'un niveau critique.

Le modèle $H A N D Y$ permettrait une meilleure compréhension des cycles historiques de croissanceeffondrement dans les interactions entre l'être humain et son écosystème. Ces oscillations présentes dans le modèle proie-prédateur sont courantes dans les systèmes naturels. Par ailleurs, "les cycles séculaires - oscillations sociopolitiques et démographiques de très longue durée (plusieurs siècles) sont la règle plutôt que l'exception dans les grands états et empires agraires" (Turchin \& Nefedov, 2009).

\footnotetext{
6 De manière schématique, dans un écosystème, lorsque la population des proies augmente, celle des prédateurs augmente également. Après un écart de temps, l'augmentation de la population des prédateurs entraîne la diminution de celle des proies, ce qui entraîne à terme la diminution de celle des prédateurs qui n'ont plus assez de nourriture. Ensuite, le cycle redémarre puisqu'avec une faible population de prédateurs, celle des proies augmente rapidement. Une succession de plusieurs cycles peut être représentée par deux sinusoïdes de populations légèrement décalées qui alternent des périodes de croissances et de déclins.
} 


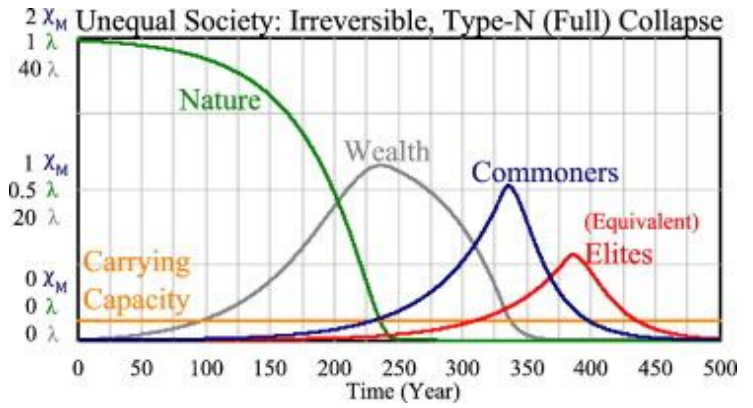

Fig.7 - Scénario de société inégalitaire : effondrement irréversible de type- $\mathrm{N}^{7}$ : un effondrement soudain et complet lié à la fois à la sur-exploitation des ressources et aux inégalités (Adapté d'après Motesharrei, Rivas \& Kalnay, 2014, fig.6b, p.98).

À partie de toutes ces études historiques et de ces modélisations d'effondrements de sociétés, anciennes ou projetées, une nouvelle science a été définie de manière controversée : la "collapsologie". Présentée par ses créateurs - les chercheurs français Pablo Servigne et Raphaël Stevens (2015) - avec "une certaine autodérision", elle serait un "exercice transdisciplinaire d'étude de l'effondrement de la civilisation industrielle et de ce qui pourrait lui succéder, en s'appuyant sur la raison, l'intuition et des travaux scientifiques reconnus". La collapsologie s'appuie sur une méthode systémique et elle prend racine dans différents travaux qui étudient l'effondrement en tant que processus complexe, nonlinéaire, ponctué par des périodes de stabilité et d'instabilité. Ainsi, ils prennent notamment appui sur le Rapport Meadows (1972), les résultats de l'International Geosphere-Biosphere Programme (IGBP) (Crutzen \& Stoermer, 2000) et les recherches menées par le chimiste américain Will Steffen et al. (2015). Ces recherches convergent avec celles de la Global History, comme dans les travaux de Laurent Testot (2017). Selon Pablo Servigne et Raphaël Stevens (2015), l'expression "effondrement de la civilisation industielle" véhicule trois clichés : un retour à la barbarie après la fin des institutions, un grand vide comme dans l'imaginaire collectif de l'apocalypse religieuse, un événement brutal qui tomberait d'un coup sur l'ensemble de la société. Mais selon eux, une définition "plus adaptée à notre temps" de l'effondrement pourrait être celle du politicien français Yves Cochet (2011) : "le processus à l'issue duquel les besoins de base (eau, alimentation, logement, habillement, énergie...) ne sont plus fournis [à un coût raisonnable] à une majorité de la population par des services encadrés par la loi".
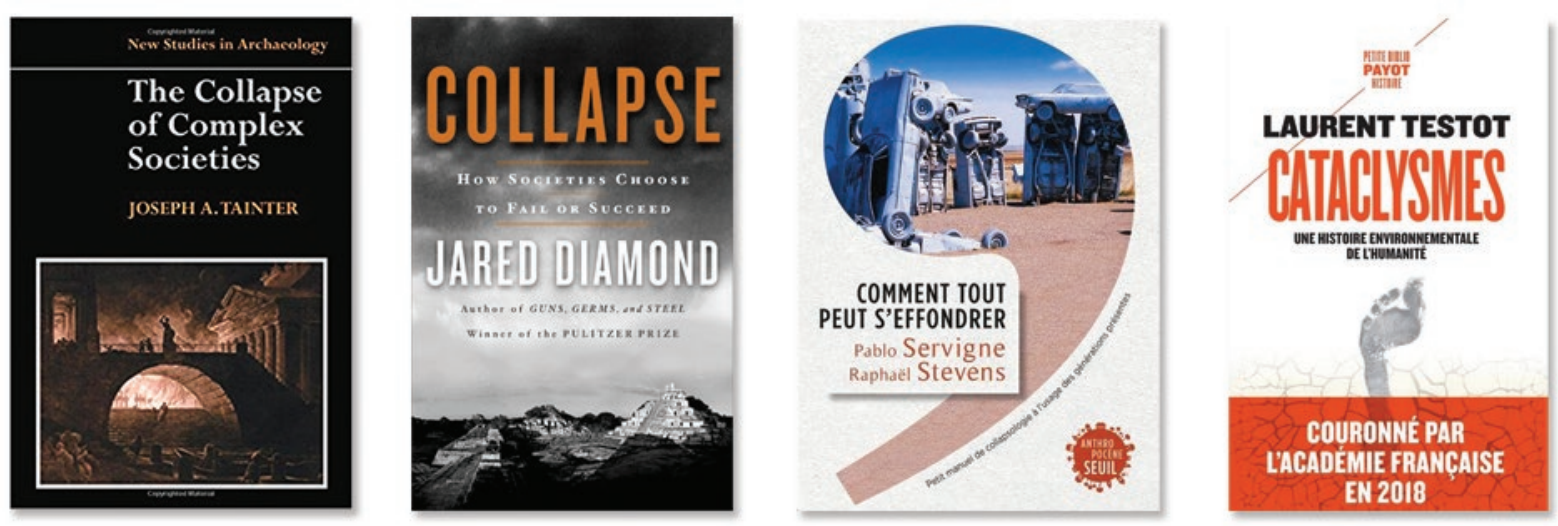

Fig. 8 - Premières de couvertures emblématiques

(Tainter, 1988 ; Diamond, 2005 ; Servigne \& Stevens, 2015 ; Testot, 2017).

\section{CONCLUSIONS}

Galvanisé par les effets du processus global d'hominisation lui laissant croire que son espèce est en mesure de dominer impunément le monde, l' "homo doublement sapiens" (Claeys, 2017) semble

\footnotetext{
7 En paramétrant le modèle HANDY à l'aide de deux types de scénarios, il produit deux types d'effondrement : le "type L" (la disparition du travail) et le "type N" (l'épuisement de la nature).
} 
oublier que - outre sa santé mentale et son insertion dans un système socioculturel - la qualité de l'environnement est la principale condition de sa survie. Par ailleurs, toutes les études de l'effondrement des sociétés passées pointent deux causes principales à cette issue funeste: l'exploitation excessive des ressources de l'environnement et la stratification abusive de la société. Quelle que soit l'époque, l'impératif de la protection environnementale et la justice sociale par la réduction des inégalités sont deux des enjeux les plus importants dont il faut rendre les exigences compatibles sous peine de voir s'effondrer toute construction sociétale. Or, en pleine ère de la capitalocène, notre société contemporaine repose sur un modèle socioéconomique exploitant sans relâche la biocapacité planétaire et amplifiant à l'extrême les inégalités sociales. De là, l'hypothèse d'un effondrement probable de nos sociétés paraît pertinente !

Toutefois, alors qu'il semblait encore impossible à mettre en œuvre à la fin du $\mathrm{XX}^{\mathrm{e}}$ siècle, un changement de paradigme pourrait finalement émerger. Des transitions s'opèrent dans les comportements et les pensées des membres de nos sociétés : de la croissance à la décroissance, de l'établissement de structures statiques à la modélisation de systèmes dynamiques, de la responsabilité face à ses propres actions à la soutenabilité pour les générations futures, de la dénonciation de l'anthropocène à celle de la capitalocène, des recherches historiques sur l'effondrement des sociétés à la conception de modèles pour en détecter les signes avant-coureur...

Face aux multiples constats alarmants annonçant un effondrement imminent de nos sociétés, les réactions les plus courantes sont le doute (ces données sont fausses, donc je ne change rien à mes habitudes), le déni (ces données n'existent pas) ou la résignation (je n'ai personnellement aucun poids, donc je ne fais rien). Pourtant cette situation angoissante peut être vue autrement : l'approche supposée de la fin du monde devrait être suffisamment stimulante pour pousser l'humanité à modifier en profondeur son organisation actuelle.

Bien que les modèles théoriques d'évolution des sociétés menacées d'effondrement présentés ici soient réducteurs et/ou trop complexes et qu'assimiler la collapsologie à une nouvelle science relève partiellement du bluff scientifique sachant que la fin du monde exercera toujours une fascination sur le public, toutes ces initiatives sont autant de d'outils théoriques et pratiques pour envisager différemment notre rapport au monde. Par ailleurs, la pensée systémique contribue largement à l'émergence de ce type de recherches.

Le mot latin humanus (humain, cultivé, civilisé) serait issu à la fois de l'humus (la terre, le sol, le terrain) - proche de humilis (qui est au sol, humble, soumis) - et de la mens (l'intelligence, la mémoire, la pensée). À la croisée de la matière et de l'esprit, l'homme sort littéralement de la terre pour émerger par la pensée. Or, pour faire honneur à l'étymologie du mot que le désigne, l'être humain doit aujourd'hui mobiliser toutes ses capacités cognitives pour rencontrer les obligations contractuelles qui le lient à Gaïa.

\section{RÉFÉRENCES}

ASSOGHA, Yao (1979). "Ivan Illich : Essai de synthèse". Critères, Montréal, n²6, pp.217-235.

BERTALANFFY, Ludwig (von) (1968). Théorie générale des systèmes. Trad. Chabrol J.-B. [General System Theory. New York: Georges Braziller]. Paris : Dunod, éd.1993.

BOULTER, Michael (2002). Extinction: Evolution and the End of Man. New York: Columbia University Press.

BRUNDTLAND, Gro Harlem (dir.) (1987). Notre avenir à tous. Trad. Luc Gagnon \& Harvey L. Mead [Our Common Future World]. Report of the World Commission on Environment and Development, The United Nations (UN). Montréal : Fleuve, éd.1989.

CLAEYS, Damien (2017). "Pour une co-conception écosystémique de l'architecture à l'ère de l'anthropocène". Roose, Marie-Clotilde (dir.) (2019). Penser à partir de l'architecture : Poétique, technique, éthique. Louvain-la-Neuve : Presses universitaires de Louvain, pp.279-308.

CLAEYS, Damien \& LAMBERT, Claude (2018). "La chute des géants : De la crise à l'émergence". Acta Europeana Systemica (AES), n8, pp.395-412. 
COCHET, Yves (2011). "L'effondrement, catabolique ou catastrophique ?". Institut Momentum, Séminiare du 27 mai 2011.

CRUTZEN, Paul J. (2002). "Geology of mankind - The Anthropocene". Nature, vol.415, n³, p.23.

CRUTZEN, Paul J. \& STOEMER, Eugène (2000). "The 'Anthropocene' ". Global Change Newsletter, $\mathrm{n}^{\circ} 41$, pp. 17-18. [The International Geosphere-Biosphere Programme (IGBP): A Study of Global Change of the International Council for Science (ICSU).]

DESCARTES, René (1637). Discours de la méthode pour bien conduire sa raison et chercher la vérité dans les sciences. Paris : Hachette (coll. Poche), éd.2000.

DIAMOND, Jared (2005). Effondrement: Comment les sociétés décident de leur disparition ou de leur survie. Trad. Agnès Botz \& Jean-Luc Fidel [Collapse: How Societies Choose to Fail ou Succed. New York: Penguin Books]. Paris : Gallimard (coll. Folio essais), éd.2006.

DION, Cyril \& LAURENT, Mélanie (2015). Demain : Partout dans le monde, des solutions existent. Move Movie, France 2 cinéma, Mars films, Mely Production.

FENNER, Franck J. (2010). "Frank Fenner sees no hope for humans". Interview accordée à The Australian, le 16 juin 2010.

FORRESTER, Jay W. (1971). World Dynamics. Cambridge, MA: Wright-Allen Press.

FORRESTER, Jay W. (1969). Urban Dynamics. Waltham, MA: Pegasus Communications.

GEORGESCU-ROEGEN, Nicholas (1979). Demain la décroissance : Entropie, écologie, économie. Trad. Jacques Grinevald \& Ivo Rens. Lausanne : Pierre-Marcel Favre.

HAMILTON, Clive \& GRINEVALD, Jacques (2015). " Was the Anthropocene anticipated?". The Anthropocene Review, vol.2, $\mathrm{n}^{\circ} 1$, pp.59-72.

HOBSBAWM, Eric J. (1975). The Age of Capital: 1848-1875. London: Weidenfeld \& Nicolson.

HOPKINS, Rob (2008). Manuel de Transition: De la dépendance au pétrole à la résilience locale. Trad. Michel Durand [The Transition Handbook: From Oil Dependency to Local Resilience. Chelsea Green Publishing]. Paris : Éditions Écosociété (coll. Guides pratiques), éd.2010.

ILLICH, Ivan (1973). La Convivialité. Paris : Seuil.

JACQUARD, Albert (2004). Tentatives de lucidité. Paris : Stock.

JONAS, Hans (1973). Le principe responsabilité : Une éthique pour la civilisation technologique. Trad. Jean Greisch [Das princip verantwortung. Insel Verlag]. Paris : Flammarion (coll. Champs), éd.2013.

LEOPOLD, Aldo (1949). Almanach d'un comté des sables. Trad. Anne Gibson [A Sand County Almanac. London: Oxford University Press]. Paris : Aubier, éd.1995.

LEWIS, S. L. \& MASLIN, M. A. (2015). "Defining the Anthropocene". Nature, n519, pp.171-180.

LOCKE, John (1690). Traité du Gouvernement Civil. Trad. David Mazel [Two Treatises of Government. London: Awnsham Churchill]. Paris : Flammarion (coll. Garnier Flammarion), éd.1999.

MALM, Andreas (2016). Fossil Capital: The Rise of Steam Power and the Roots of Global Warming. London: Verso Books.

MARSH, George P. (1864). Man and Nature, or Physical Geography as Modified by Human Action. New York: Charles Scribner, $2^{\mathrm{e}}$ édition augmentée en 1885.

MATURANA, Humberto R. \& VARELA, Francisco J. (1972). "Autopoiesis: The Organization of the Living". Trad. Maturana H.R. [De Máquinas y Seres Vivos]. Autopoiesis and Cognition: The Realization of the Living. Dordrecht: Kluwer Academic Publishers, éd.1980, pp.59-138.

MEADOWS, Dennis L. (2013). "Il est trop tard pour le développement durable". SINAÏ, Agnès (dir.) (2013). Penser la décroissance: Politiques de l'anthropocène. Les presses de Science-Po (coll. Nouveaux débats), pp.195-210. 
MEADOWS, Donella H., MEADOWS, Dennis L., RANDERS, Jørgen (2004). Les limites à la croissance (dans un monde fini) : Le Rapport Meadows, 30 ans après. Trad. Agnès El Kaïm [The Limits to Growth: the 30-Year Update. New York: Universe Books]. Paris : Fayard.

MEADOWS, Donella H., MEADOWS, Dennis L., RANDERS, Jørgen \& BEHRENS III, W. W. (1972). Halte à la croissance? Trad. Jacques Delaunay [The Limits to Growth: A report for The Club of Rome's project on the predicament of mankind. New York: Universe Books]. Paris : Fayard.

MORIN, Edgar (1973). Le paradigme perdu : La nature humaine. Paris : Seuil (coll. Points).

MORIN, Edgar (2001). La méthode 5 : L'humanité de l'humanité. L'identité humaine. Paris : Seuil (coll. Points).

MORIN, Edgar (2011). La Voie: Pour l'avenir de l'humanité. Paris: Fayard (coll. Sciences humaines).

MOTESHARREI, Safa, RIVAS, Jorge, KALNAY, Eugenia (2014). "Human and nature dynamics (HANDY): Modeling inequality and use of resources in the collapse or sustainability of societies". Ecological Economics, vol.101, mai 2014, pp.90-102.

ORLOV, Dmitry (2013). Les cinq stades de l'effondrement. Trad. Tancrède Bastié [The Five Stages of Collapse: Survivors' Toolkit. New Society Publishers]. Le Retour aux Sources, éd.2016.

REGGIO, Godfrey (1982). Koyaanisqatsi: Life Out of Balance. IRE Productions, Santa Fe Institute for Regional Education.

REVKIN, Andrew C. (1992). Global Warming: Understanding the Forecast. New York: Abbeville Press.

ROUSSEAU, Jean-Jacques (1762). Du contrat social ou Principes du droit politique. Amsterdam: Marc Michel Rey.

SADIN, Éric (2016). La silicolonisation du monde: L'irrésistible expansion du libéralisme numérique. Montreuil : L'échappée (coll. Pour en finir avec).

SERVIGNE, Pablo \& STEVENS, Raphaël (2015). Comment tout peut s'effondrer : Petit manuel de collapsologie à l'usage des générations présentes. Paris : Seuil (coll. Anthropocène).

SMITH, Adam (1759). Théorie des sentiments moraux. Trad. Michaël Biziou, Claude Gautier et JeanFrançois Pradeau [The Theory of Moral Sentiments. London]. Paris : PUF (coll. Quadrige), éd.2014.

STEFFEN, Will, SANDERSON Angelina, TYSON, Peter D. et al. (2004). Global Change and the Earth System: A Planet Under Pressure. The IGBP Book Series. Berlin, Heidelberg, New York: Springer-Verlag.

STEFFEN, Will et al. [Broadgate, Wendy, Deutsch, Lisa, Gaffney, Owen, Ludwig, Cornelia] (2015a). "The Trajectory of the Anthropocène: The Great Acceleration". The Anthropocène Review, vol.2, n 1 , pp.81-98.

STEFFEN, Will et al. (2015b). "Planetary Boundaries: Guiding human development on a changing planet". Science, vol.347, n6223, pp.736-746.

STOPPANI, A (1873). Corso di Geologia: vol. II, Geologia stratigrafica. Milano: G. Bernardoni E. G. Brigola.

TAINTER, Joseph A. (1988). L'Effondrement des sociétés complexes. Trad. Jean-François Goulon [The Collapse of Complex Societies. Cambridge University Press]. La Fenderie : Le Retour aux sources, éd.2013.

TANSLEY, Arthur G. (1935). "The Use and Abuse of Vegetational Concepts and Terms". Ecology, vol.16, n³, pp.284-307.

TESTOT, Laurent (2017). Cataclysmes : Une histoire environnementale de l'humanité. Paris : Payot \& Rivages.

TEILHARD DE CHARDIN, Pierre (1955). Le phénomène humain. Paris : Seuil, éd.2007.

TURCHIN, Peter \& NEFEDOV, Sergey A. (2009). Secular Cycles. Princeton University Press. 
TURNER, Graham. M. (2012). "On the cusp of global collapse? Updated comparison of the Limits to Growth with historical data". GAiA - Ecological Perspectives for Science and Society, vol.21, n², pp.116-124.

VERNADSKI, Vladimir I. (1926). La Biosphère. Trad. depuis le russe [Biosfera. Leningrad]. Paris : Librairie Félix Alcan, éd.1929.

VERNADSKI, Vladimir I. (1945). "The Biosphere and the Noosphere". American Scientist, vol.33, $\mathrm{n}^{\circ} 1, \mathrm{pp} .1-12$. 